\title{
Morfología y fenología floral de Nothofagus alpina (Nothofagaceae) en un huerto semillero clonal en la región de Los Ríos, Chile
}

\author{
Floral morphology and phenology of Nothofagus alpina (Nothofagaceae) in a clonal \\ seed orchard in Los Ríos Region, Chile
}

\section{Luz García a*, Fernando Droppelmann ${ }^{b}$, Magaly Rivero ${ }^{c}$}

*Autor de correspondencia: ${ }^{a}$ Universidad Austral de Chile, Facultad de Ciencias Forestales y Recursos Naturales, Programa de Doctorado en Ciencias Forestales, Valdivia, Chile, luzcecilia29@hotmail.com

${ }^{\mathrm{b}}$ Universidad Austral de Chile, Facultad de Ciencias Forestales y Recursos Naturales, Valdivia, Chile.

${ }^{\mathrm{c}}$ Cooperativa de Mejoramiento Genético de Chile, Valdivia, Chile.

\section{SUMMARY}

Reproductive biology knowledge of species is essential for in situ conservation, propagation and breeding. Consequently, we describe the first detailed phenology of Nothofagus alpina, mentioned until now only in general terms, and provide the morphological description of the male and female flowers. The study was conducted in 2011 in the Clonal Seed Orchard "Huillilemu" located in the locality of San José Mariquina, Region Los Ríos, Chile, at 23 meters of altitude. Observations were made in six clones of the orchard. The phenology model proposed presents ten states from the leaf bud in latency to the initial fruit. The first phenophase characterizes the leaf bud state in rest, while the second shows the morphological changes associated with getting out of dormancy. Five states described below develop male flowers and female inflorescences that appear later. The fall of male flowers mark the transition status into the female flower. The last two phenological stages describe the initial infructescence and development of the dome, respectively. These changes took place over a period of 58 days in individuals with early budding and in 40 days in late budding individuals with accumulated heat requirement of 560-623 GDD. The start date break was related to the altitudinal origin of trees, suggesting a genetic control of this feature. The overall overlap rate of flowering was calculated by 0.22 , indicating low reproductive synchrony among analyzed individuals.

Key words: characterization morphology, reproduction, dichogamy, overlapping index, flowering.

\section{RESUMEN}

El conocimiento de la biología reproductiva de las especies, es fundamental para la conservación in situ, reproducción y mejoramiento genético. Por ello, se describe por primera vez la fenología detallada de Nothofagus alpina, hasta ahora mencionada sólo de forma general y se presenta la descripción morfológica de las flores masculinas y femeninas. El estudio se realizó durante el año 2011, en el huerto semillero clonal "Huillilemu", ubicado en la comuna San José de la Mariquina, región de Los Ríos, Chile, a una altitud de $23 \mathrm{~m}$ snm. Las observaciones se realizaron en seis clones del huerto. El modelo fenológico propuesto, presenta 10 estados, desde la yema en latencia hasta el fruto inicial. El primer estado caracteriza a la yema en reposo, mientras que el segundo, ilustra los cambios morfológicos asociados a la salida del reposo. Cinco estados describen a continuación, el desarrollo de las flores masculinas y de las inflorescencias femeninas, que aparecen después de estas. La caída de las flores masculinas, marca en la flor femenina la transición al estado de fruto. Los dos últimos estados fenológicos, describen la infrutescencia inicial y desarrollo de la cúpula, respectivamente. Estos cambios se desarrollaron durante un lapso de 58 días, en los individuos con brotación temprana y en 40 días en los individuos con brotación tardía, con requerimiento de calor acumulado de 560 a 623 grados días de desarrollo. La fecha de inicio de la brotación, está relacionada con la procedencia altitudinal de los árboles, por lo que se sugiere un control genético de esta característica. El índice de sobreposición general de la floración se calculó en 0,22, indicando una baja sincronía reproductiva entre los individuos analizados.

Palabras clave: caracterización morfológica, reproducción, dicogamia, índice de sobreposición, floración.

\section{INTRODUCCIÓN}

Nothofagus alpina (P. et E.) Oerst. es una especie endémica de los bosques templados subantárticos, que pierde sus hojas estacionalmente. Se distribuye en el extremo suroccidental de Sudamérica, en territorio chileno y argentino (Donoso et al. 2006). Botánicamente N. alpina es una es- pecie dicotiledónea, monoica, anemófila; perteneciente a la familia monogenérica Nothofagaceae (Hill y Jordan 1993).

El conocimiento de la biología reproductiva de las especies, es fundamental para la conservación in situ, reproducción y mejoramiento genético, pero la escasa información en la mayoría de las especies forestales, dificulta el inicio de los trabajos para tales fines. Es primordial contar 
con información detallada de las características reproductivas de las especies, incluido el desarrollo de los órganos reproductivos en el tiempo, es decir su fenología.

La literatura científica no registra trabajos referentes a la anatomía de los órganos reproductivos en especies del género Nothofagus, siendo muy pocos los referentes a la morfología; pudiendo citarse a Urban (1934), quien describe la morfología de las flores de algunos Nothofagus; Rozefelds y Drinnan $(1998,2002)$ que estudiaron la ontogenia y diversidad de las flores masculinas y femeninas de varias especies del género Nothofagus. Así mismo, algunos trabajos analizan la fenología estacional de especies de Nothofagus (Rusch 1993, Premoli et al. 2007), pero se requiere la fenología detallada, para la toma de decisiones en los procesos de mejoramiento y manejo forestal.

La fenología contempla el estudio de los fenómenos periódicos de los vegetales y la relación de estos eventos con factores edáficos y climáticos (De Fina y Ravelo 1979, Martí et al. 2004). Según Lieth (1997) la fenología clásica es el arte de subdividir el desarrollo de taxas biológicos, en secciones identificables a lo largo de un tiempo. Estas secciones se denominan fenofases y se utilizan para examinar el desarrollo del ciclo de vida de un organismo. La ocurrencia de una fenofase, es determinada por las características bióticas de las especies vegetales y por las características del clima local.

En los climas templados la secuencia anual de fenofases de las plantas leñosas, está claramente relacionada con la estacionalidad del clima, principalmente con la variación de la temperatura y el fotoperiodo. Ello sugiere que las fenofases deben producirse en el curso del año, en cada individuo con perfecta coordinación entre ellas y que el patrón fenológico debe estar suficientemente adaptado a las condiciones ambientales, para asegurar la supervivencia de la planta (Martí et al. 2004).

Esta ciencia ha emergido recientemente como objetivo importante de la investigación ecológica (Schwartz 1999) y es de gran valor en otras áreas de investigación: agricultura, salud pública, ecología, fitoclimatología. En las ciencias forestales su estudio puede ayudar al manejo adecuado de los cultivos, apoyando al establecimiento de modelos de producción, nutrición; determinar posibles anomalías de tipo fisiológico, establecer la época adecuada para realizar cruzamientos controlados y recolección de semillas.

En vista de que la fenología sienta las bases para comprender la biología reproductiva de las especies, se pretende iniciar el estudio de la biología floral de $N$. alpina con un análisis de la fenología floral. En la presente investigación, se planteó realizar una descripción morfológica de las flores de $N$. alpina, establecer un patrón fenológico de floración, y determinar la longevidad floral de seis genotipos de $N$. alpina de diferentes orígenes altitudinales; conocimiento necesario para la planificación de cruzamientos en los programas de mejoramiento genético y para la producción de semillas mejoradas, para abastecer los programas de reforestación con esta especie.

\section{MÉTODOS}

El estudio se realizó durante el año 2011, en el huerto semillero clonal Huillilemu, ubicado en la comuna San José de la Mariquina, región de Los Ríos, Chile, a una altitud de $23 \mathrm{~m}$ snm. Según la clasificación de Köppen (1900), esta región presenta un clima templado lluvioso; se clasifica dentro del subtipo clima de costa occidental con influencia mediterránea y se caracteriza por las altas precipitaciones, con un promedio anual de $2.400 \mathrm{~mm}$. Las condiciones climáticas durante el tiempo en que se desarrolló el estudio, se detallan en la figura 1 (Sistema Agroclimático 2012). El calor acumulado (figura 1) se calculó en unidades grados días de desarrollo, desde el 01 de julio al 25 de noviembre del 2011, utilizando la fórmula 1 (De Fina y Ravelo 1979):

$\mathrm{GD}=\frac{(\mathrm{Tmax}+\mathrm{Tmin})}{2}-\mu$

Donde:

$\mathrm{GD}$ = grados días $\left({ }^{\circ} \mathrm{C}\right)$,

Tmax $=$ temperatura máxima $\left({ }^{\circ} \mathrm{C}\right)$,

Tmin $=$ temperatura mínima $\left({ }^{\circ} \mathrm{C}\right)$,

$\mu=$ temperatura umbral mínima de actividad fisiológica $\left({ }^{\circ} \mathrm{C}\right)$.

La fórmula 1 pertenece al método residual, donde $\mu$ corresponde a la temperatura umbral mínima de actividad. Para los cálculos correspondientes se utilizó $\mu=7^{\circ} \mathrm{C}$, por ser considerada como limitante para la actividad fisiológica de los árboles; el desarrollo de la mayoría de las especies agrícolas y forestales ocurre sobre ésta temperatura (De Fina y Ravelo 1979).

El estudio morfológico se realizó sobre material colectado y se complementó con observaciones del periodo de floración de la especie; definiendo como floración el desarrollo de las flores, desde el momento de la antesis, hasta la fase de senescencia (Souza et al. 2003). Para determinar el periodo de vida; en las flores masculinas se consideró su duración en días, desde el estado de flor visible hasta su senescencia; la duración de las flores femeninas se calculó desde la fecha que fueron visibles a ojo desnudo, hasta la etapa de flor madura o fruto inicial. Las visitas al huerto para las respectivas observaciones, se realizaron trisemanalmente, durante las cuales se efectuó un registro fotográfico de los estados de las flores en cada una de las ramas marcadas previamente.

Las observaciones fenológicas se realizaron en seis clones, de dos a cinco rametos por clon, plantados en el año 1989. En cada árbol se etiquetaron cinco ramas, en diferentes orientaciones, que tenían entre 8 y 12 brotes. En adelante, se denominará unidades estructurales de crecimiento (UEC), a la nueva ramilla desarrollada a partir de un evento de extensión ininterrumpida o elongación de un brote (Puntieri et al. 2007). Los individuos evaluados se detallan en la cuadro 1.

Para determinar el fenoestado de las UEC, se siguió la siguiente pauta: 


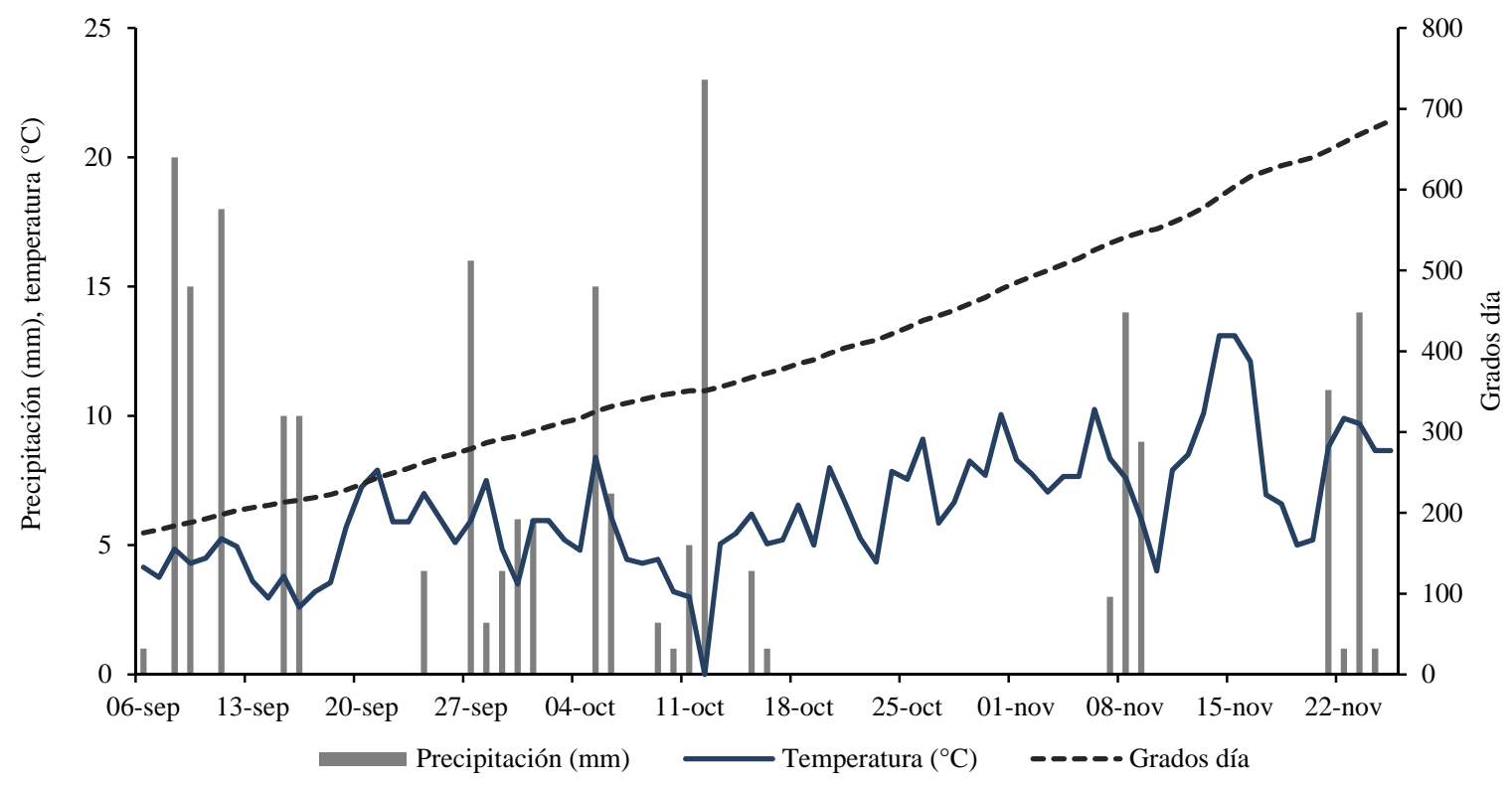

Figura 1. Condiciones climáticas en el huerto semillero Huillilemu, 2011.

Climatic conditions in the seed orchard Huillilemu, 2011.

Cuadro 1. Código y origen de los árboles evaluados. Code and origin of evaluated trees.

\begin{tabular}{ccccc}
\hline Código & $\begin{array}{c}\text { Lugar de } \\
\text { origen }\end{array}$ & $\begin{array}{c}\text { Altitud } \\
(\mathrm{m} \mathrm{snm})\end{array}$ & Latitud S & Longitud O \\
\hline 38 & Arquilhue & 540 & $40^{\circ} 07^{\prime}$ & $72^{\circ} 02^{\prime}$ \\
11 & Neltume & 620 & $39^{\circ} 49^{\prime}$ & $71^{\circ} 58^{\prime}$ \\
361 & Arquilhue & 670 & $40^{\circ} 07^{\prime}$ & $71^{\circ} 55^{\prime}$ \\
19 & Neltume & 700 & $39^{\circ} 51^{\prime}$ & $71^{\circ} 53^{\prime}$ \\
4 & Pilmaiquén & 1.000 & $39^{\circ} 58^{\prime}$ & $71^{\circ} 48^{\prime}$ \\
30 & Pilmaiquén & 1.000 & $39^{\circ} 54^{\prime}$ & $71^{\circ} 51^{\prime}$ \\
\hline
\end{tabular}

- Se consideró como inicio de apertura de yemas florales, cuando al menos el $20 \%$ de los brotes en las ramas observadas alcanzó dicha fase.

- Se determinó las fases de senescencia y caída de las flores masculinas, cuando se observó en el 80 \% o más de los brotes en las ramas evaluadas.

- El resto de las fases fenológicas registradas, se determinaron cuando el $50 \%$ o más de los brotes en las ramas observadas alcanzó dicha fase (plenitud de fase).

Para determinar la fenofase en que se encontraba cada árbol, se le asignó el estado fenológico registrado para la mitad o más de sus UEC evaluadas (Gómez-Casero et al. 2007). La designación del estado fenológico, se hizo mediante la visualización directa de las características de cada órgano.

A partir de los datos obtenidos, se diseñó un modelo conjunto (cuadro 2, figura 2) donde se establecen los es- tados fenológicos para las UEC, integrando las fenofases presentadas por las flores masculinas y femeninas. Las fenofases se designaron con una letra continua del alfabeto, por ejemplo: A para la yema cerrada, B para la yema hinchada, y así hasta el estado final de la variable fenológica. Para nominar las fases fenológicas de las flores masculinas y femeninas, se les asignó un número en una escala ordinal. En vista de que en una misma UEC, se encuentran tanto hojas, como las flores masculinas y femeninas, también se realizó un registro del desarrollo de las hojas, aunque este no fue decisivo en la elaboración del patrón fenológico, puesto que tanto el tamaño como el número de hojas en los diferentes brotes, en los árboles evaluados, presentaban una gran variación dentro de una misma fenofase.

Se determinó la sobreposición del periodo de floración, según el índice cualitativo de Sörensen (1948), utilizando la fórmula 2:

$$
\mathrm{S}=\frac{2 c}{a+b}
$$

Donde:

$a=$ fechas de floración masculina del individuo $x$,

$b=$ fechas de floración femenina del individuo $y$,

$c=$ fechas comunes de floración.

Para el cálculo, sólo se consideraron las etapas de liberación de polen en las flores masculinas y receptividad del estigma en las flores femeninas. Según este índice, un valor igual o cercano a 1 indica un elevado solapamiento de la floración entre los individuos comparados; valores cercanos a 0 son indicativos de un bajo grado de solapamiento entre los individuos. 
Cuadro 2. Estados fenológicos de la foliación y desarrollo de flores masculinas y femeninas. Phenological stages of the foliation and development of male and female flowers.

\begin{tabular}{|c|c|c|c|c|c|c|}
\hline Estado UEC & \multicolumn{2}{|c|}{ Foliación } & \multicolumn{2}{|c|}{ Flores masculinas } & \multicolumn{2}{|c|}{ Flores femeninas } \\
\hline A & 1 & Yemas cerradas & & - & & - \\
\hline B1 & 2 & Yema hinchada, ápice verde & & - & & - \\
\hline B2 & 3 & Inicio de apertura de yemas & 1 & Flor visible & & - \\
\hline $\mathrm{C}$ & 4 & $\begin{array}{l}\text { Despliegue de primeras } \\
\text { hojas (1-4 hojas) }\end{array}$ & 2 & $\begin{array}{l}\text { Flor expuesta } \\
\text { completamente }\end{array}$ & & - \\
\hline D1 & 5 & $\begin{array}{l}\text { Despliegue de hojas } \\
\text { (5-6 hojas) }\end{array}$ & 3 & $\begin{array}{l}\text { Flor en proceso de } \\
\text { maduración de anteras }\end{array}$ & 1 & Inflorescencia visible \\
\hline $\mathrm{E}$ & 6 & $\begin{array}{l}\text { Despliegue de hojas } \\
(+6 \text { hojas })\end{array}$ & 4 & Anteras maduras. & 2 & $\begin{array}{l}\text { Inflorescencias que presentan } \\
\text { estigmas en crecimiento }\end{array}$ \\
\hline $\mathrm{F}$ & & - & 5 & Liberación de polen & 2 & $\begin{array}{l}\text { Estigmas de flores en } \\
\text { crecimiento }\end{array}$ \\
\hline G & & - & 6 & Anteras vacías. Senescencia & 3 & Flores con estigmas receptivos \\
\hline H1 & & - & 7 & Caída de flores & 4 & $\begin{array}{l}\text { Desarrollo de valvas encerrando } \\
\text { los estigmas e inicio de } \\
\text { hinchamiento de ovarios }\end{array}$ \\
\hline $\mathrm{H} 2$ & & - & & - & 5 & Estigmas encerrados por valvas \\
\hline I & & - & & - & 6 & Desarrollo de ovarios \\
\hline $\mathrm{J}$ & 7 & $\begin{array}{l}\text { Hojas completamente } \\
\text { desplegadas }\end{array}$ & & - & 7 & Frutos tiernos \\
\hline
\end{tabular}

\section{RESULTADOS}

Caracterización de las flores. Esta especie es diclino monoica, es decir, produce flores unisexuales pistiladas y estaminadas en un mismo árbol e incluso en el mismo brote. Las flores que emergen en un periodo de floración, están preformadas desde la estación de crecimiento anterior. Se observaron brotes florales, preformados desde el final de la primavera anterior al periodo de floración respectivo. Nothofagus alpina presenta dicogamia interfloral en la modalidad de protandria, pues presenta primeramente la maduración de las flores masculinas; mientras la receptividad de los estigmas en las inflorescencias femeninas, sólo se observa al final de la etapa de liberación de polen.

Flores masculinas. Las flores masculinas son solitarias. Se encuentran en la base de las axilas de las dos hojas más basales, en número de una a cinco (por axila). Estas flores están sostenidas por un pedúnculo delgado de 1 a 1,4 cm de largo aproximadamente, característica que facilita su movimiento y la dispersión del polen por el viento, formadas por 45,7 ( $\pm 5,8)$ estambres (cuadro 3 ), que están rodeados por un perigonio verdoso, membranoso, aterciopelado, de forma semiesférica y de borde levemente aserrado. Los estambres son exertos, pubescentes, libres; las anteras son ditecas y basifijas; presentan dehiscencia longitudinal y producen una gran cantidad de polen seco amarillento; los filamentos $(0,35 \pm 0,1 \mathrm{~cm})$ son levemente más largos que las anteras $(0,28 \pm 0,1 \mathrm{~cm})$.
Cuadro 3. Cantidad de anteras en flores masculinas de Nothofagus alpina.

\begin{tabular}{rrrrrrr}
\multicolumn{6}{c}{ Number of anthers in male flowers of Nothofagus alpine. } \\
\hline \multirow{5}{*}{ Flores } & \multicolumn{5}{c}{ Código de individuos } \\
\cline { 2 - 7 } & clon 38 & clon 11 & clon 361 & clon 19 & clon 4 & clon 30 \\
\hline 1 & 50 & 53 & 56 & 45 & 36 & 54 \\
2 & 42 & 50 & 51 & 42 & 27 & 49 \\
3 & 45 & 46 & 46 & 48 & 33 & 51 \\
4 & 49 & 57 & 46 & 47 & 32 & 51 \\
5 & 45 & 43 & 40 & 52 & 33 & 53 \\
6 & 47 & 50 & 48 & 48 & 39 & 48 \\
7 & 48 & 38 & 56 & 50 & 42 & 48 \\
8 & 59 & 44 & 42 & 48 & 35 & 48 \\
9 & 49 & 45 & 40 & 40 & 34 & 49 \\
10 & 52 & 45 & 46 & 48 & 31 & 54 \\
$\bar{y}$ & 48,6 & 47,1 & 47,1 & 46,8 & 34,2 & 50,5 \\
Sd & 4,64 & 5,47 & 5,82 & 3,58 & 4,18 & 2,46 \\
\hline
\end{tabular}

Flores femeninas. Las flores son verdes, desnudas e inconspicuas; se encuentran en inflorescencias constituidas por tres flores de grandes estigmas, envueltas en un invólucro común, que consiste en un conjunto de valvas, que en la madurez se convierte en la cúpula tetrapartida que encierra los frutos. Las inflorescencias femeninas se encuentran, entre las estípulas, en las axilas de las hojas más 
I

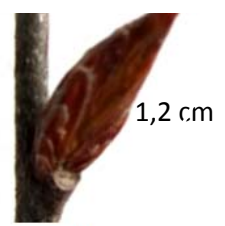

A

II
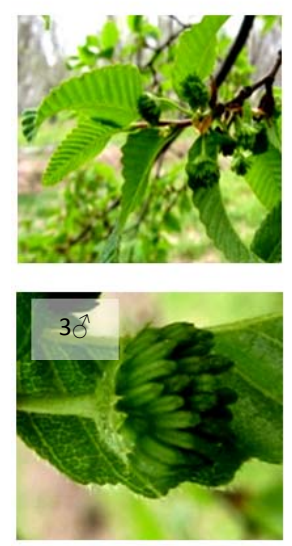

IV

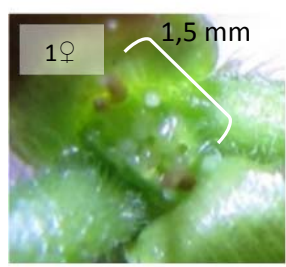

D

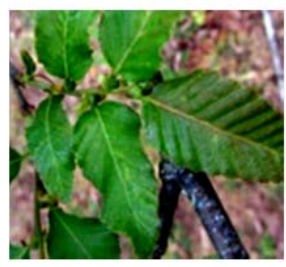

VI

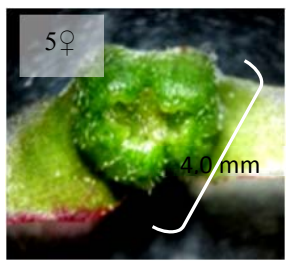

$\mathrm{H} 2$

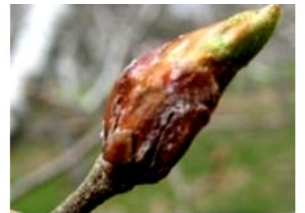

B1
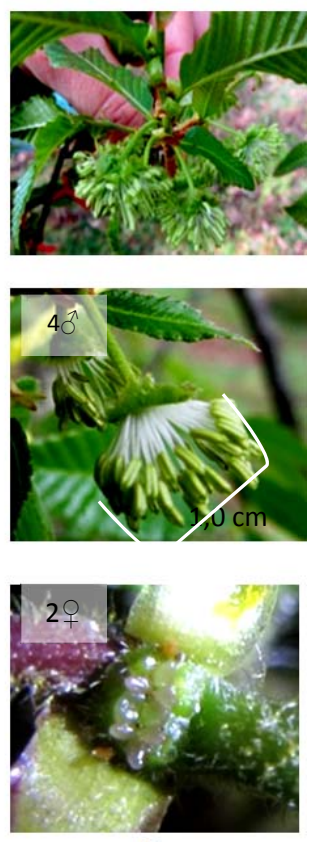

E
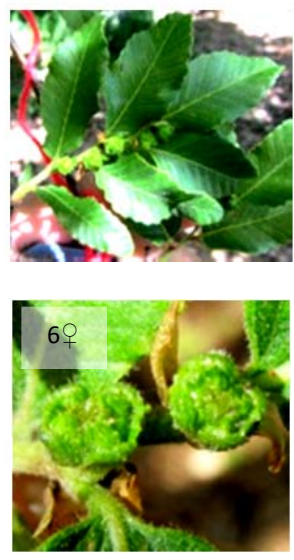

I1

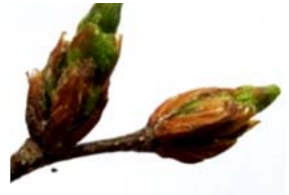

B2
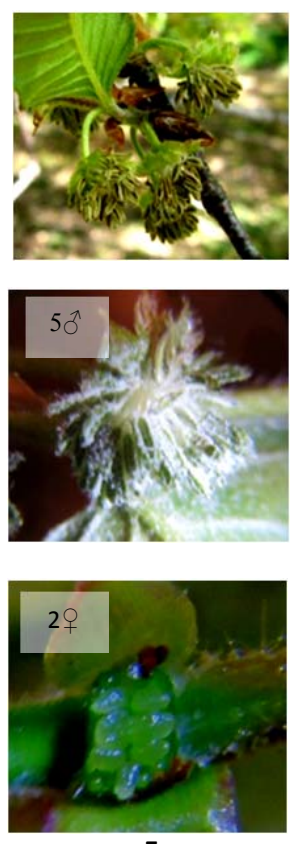

F
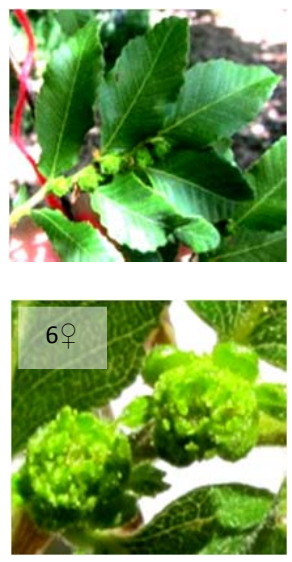

12

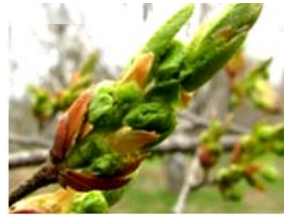

B3
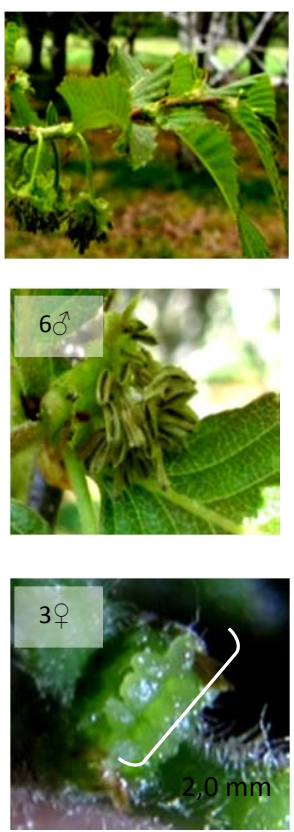

G
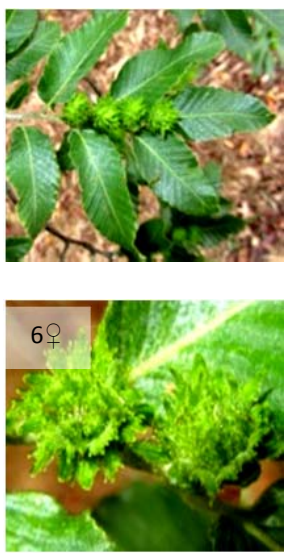

13

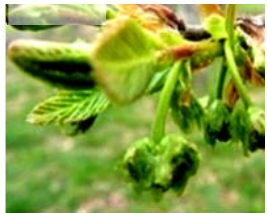

C
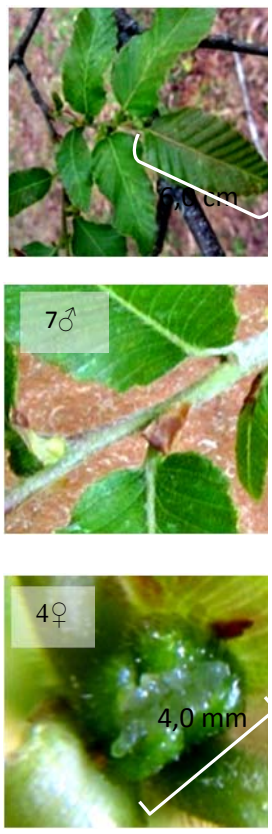

H1
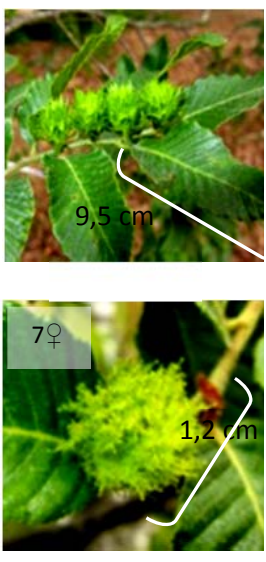

J

Figura 2. Patrón fenológico de la floración de Nothofagus alpina (A = yema en latencia, B1-B3 = yema hinchada, inicio de apertura de yema - flores masculinas visibles $(1 \hat{\jmath}), \mathrm{C}=$ flor masculina expuesta $(2 \hat{\jmath}), \mathrm{D}=$ anteras en proceso de maduración $(3 \hat{\jmath})$ - inflorescencia femenina visible $(1+$ ), $\mathrm{E}=$ anteras maduras $(4 \hat{\delta})$ y estigmas de flores femeninas en crecimiento $(2$ q $)$, $\mathrm{F}=$ liberación de polen $(5 \hat{\jmath})$ - estigmas de flores femeninas en crecimiento $(2 \circ), \mathrm{G}=$ flor masculina senescente con anteras vacías $(6 \hat{\jmath})$ - flores femeninas con estigmas receptivos $(3+), \mathrm{H} 1-\mathrm{H} 2=$ caída de flores masculinas $(7 \mathrm{~d})$-crecimiento de valvas encerrando a los estigmas $(4+9,5+)$, I1-I3 = hinchamiento de ovarios $(6$ ㅇ), $\mathrm{J}=$ fruto tierno (7ㅇ). Línea I desde yema en latencia hasta flor masculina expuesta (A-C); líneas III y IV son ampliaciones de las flores masculinas y femeninas, respectivamente, de brotes en línea II; VI corresponde a flores femeninas ampliadas de brotes en línea V.

Phenological pattern of flowering of Nothofagus alpine. A = Bud latency, B1-B3 = swollen bud, bud aperture start - male flowers visible

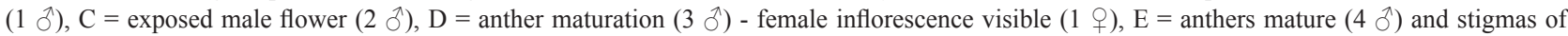
female flowers growing ( 2 \%), $\mathrm{F}=$ liberation pollen $(5 \hat{\jmath})$ - stigmata of growing female flowers $(2+), \mathrm{G}=$ senescent male flower with empty anthers

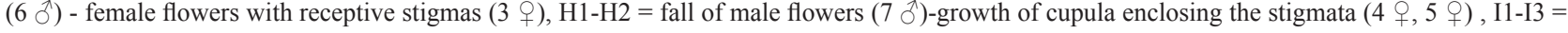
enlargement of ovaries ( 6 \%), $\mathrm{J}=$ tender fruit (7 9 ). Line I from bud dormant until exposed male flower (AC) lines III and IV are extensions of the male and female flowers, respectively, online outbreak II, VI corresponds to female flowers buds expanded online V. 
apicales, en número de una a cinco por brote (según lo registrado durante esta investigación, aunque los autores han realizado observaciones de árboles de $N$. alpina, plantados en otros lugares con 10 a 12 inflorescencias por brote). Se sostienen por pedúnculos muy cortos, casi imperceptibles, que se alargan cuando la flor madura y empieza la transición a fruto. Los estigmas sésiles, grandes y sobresalientes, se aprecian levemente mucilaginosos en la fase receptiva. La flor central tiene dos estigmas (bicarpelar) y las flores laterales tienen tres estigmas (tricarpelar). La inflorescencia en estado de receptividad tiene un tamaño de $0,22 \pm 0,02 \mathrm{~cm}$, y la infrutescencia alcanza $4,5 \pm 0,5 \mathrm{~cm}$. Los ovarios tienen la misma forma que tendrá las semillas, siendo bialados o trialados, según sean de flores centrales o laterales, con una placentación de tipo apical, y uno o dos óvulos, en cuyo caso uno degenera, quedando solo uno que será fertilizado y formará el embrión.

Fenología de la floración, caracterización morfológica de los estadios. Al mismo tiempo que se inicia la apertura de los brotes florales, se presentan las flores masculinas y más tarde las femeninas, en una misma unidad estructural de crecimiento, razón por la que se realizó un patrón fenológico conjunto. Además, porque este tipo de modelo fenológico tendría la facilidad operativa de poder determinar el estado y ubicación de las flores masculinas y femeninas (diminutas) a partir de un mismo brote. En este patrón fenológico, los cambios progresivos desde la yema latente, hasta la presencia de la infrutescencia inicial, se resumen en 10 estados morfológicos (figura 2). El modelo propuesto se describe a continuación:

- Estado A - yema en latencia. Esta fase dura desde la generación de las yemas, hasta su apertura, en el periodo vegetativo siguiente al de su formación. Las yemas se muestran cerradas, son de forma aguda, de color marrón-claro, glabras, cubiertas por brácteas coriáceas. Estas yemas se encuentran en las axilas de las hojas del ciclo vegetativo anterior y pueden ser terminales o axilares. Miden de 0,6 a 1,4 cm de longitud (figura 2A).

- Estado B - yema hinchada. Durante este estudio, esta fase duró de cuatro a ocho días (cuadro 4). Como consecuencia del despertar vegetativo, las yemas se hinchan y luego se comienza a elongar el brote observándose un ápice verde; simultáneamente se produce la apertura de las brácteas, dejando al descubierto las hojas, que todavía no han comenzado su extensión. También se pueden observar las estructuras florares masculinas, en el caso de yemas florales (figuras 2B1, 2B2, 2B3).

- Estado C - flor masculina expuesta. Durante este estudio, esta fase duró de cuatro a siete días (cuadro 4). La elongación de los entrenudos y pedúnculos sucede rápidamente, dejando al descubierto estructuras foliares y florales. Las primeras hojas aparecen al mismo tiempo que las flores masculinas y se elongan rápidamente, son pequeñas, de color verde claro. La flor masculina nace en las axilas de las dos primeras hojas más basales. Se ubican en la base de las estípulas, en número de una a cinco flores por axila. Estas flores, en esta fase, presentan las anteras encerradas por un perigonio verdoso y pubescente; el pedúnculo es delgado, con una longitud de 1,0 a 1,4 cm (figura 2C).

- Estado D - periodo de maduración de anteras- inflorescencia femenina visible. En este periodo las anteras comienzan a aparecer a través del perigonio, debido a la elongación del filamento que llega a medir hasta un $60 \%$ del total de la longitud del estambre. En esta fase las inflorescencias femeninas son visibles, en la axila de las hojas apicales, con ayuda de una lupa de bolsillo (figura 2D).

- Estado E- anteras maduras - estigmas de flores femeninas en crecimiento. En este estado, las flores masculinas se presentan con los estambres separados, que miden de 7 a $10 \mathrm{~mm}$ de longitud. Las anteras de color verde claro, están sostenidas por delgados, pero largos, flexibles y resistentes filamentos. En esta fenofase, las inflorescencias femeninas son visibles a ojo desnudo, nacen en las axilas de las hojas más apicales del brote, una inflorescencia por axila, y de una a cinco por brote. Estas inflorescencias constan de tres flores incompletas e inconspicuas, que presentan los estigmas descubiertos. Se distinguen ocho estigmas incipientes, tres por cada una de las flores laterales y dos estigmas que corresponden a la flor central. Los estigmas de flores femeninas se observan relativamente grandes y turgentes. En algunos casos, es posible observar una coloración violácea en los estigmas (figura 2E).

- Estado F- liberación de polen -estigmas de flores femeninas en crecimiento. Durante este estudio, esta fase duró de cuatro a ocho días (cuadro 4). Las anteras se abren longitudinalmente liberando el polen, la dehiscencia es extrorsa, característico de las especies anemófilas. Al mismo tiempo la flor femenina presenta los estigmas progresivamente más grandes (figura $2 \mathrm{~F}$ ).

- Estado G- flor masculina con anteras vacías - flores femeninas con estigmas receptivos. En este estado, las flores masculinas presentan un color grisáceo oscuro, con estambres vacíos; esta fase fenológica ocurre al final de la liberación del polen y se prolonga durante la caída paulatina de las flores senescentes (cuadro 4). En las flores femeninas, se observan los estigmas receptivos, con aspecto hinchado y ligeramente mucilaginoso; esta fenofase se presentó, durante un día, inmediatamente después que las flores masculinas habían completado la etapa de liberación de polen (figura $2 \mathrm{G}$, cuadro 4).

- Estado H- senescencia y caída de flores masculinas -desarrollo de valvas encerrando a los estigmas. La caída de las flores masculinas sucedió durante uno a ocho días en los diferentes genotipos (cuadro 4). En este periodo, las flores masculinas vacías y senescentes, que presentan un color plomizo claro, caen paulatinamente, quedando solamente las flores femeninas. En 
Cuadro 4. Inicio de brotación, dicogamia y duración de las fenofases en la floración de Nothofagus alpina, 2011. Se indica floración masculina y femenina *, L p: liberación de polen $\mathrm{R}$ : flor femenina receptiva.

Beginning of leaf bud breaking, dichogamy and duration of flowering phenophases in Nothofagus alpina 2011. It shows male and female flowering*, L p: R pollen release: receptive female flower.

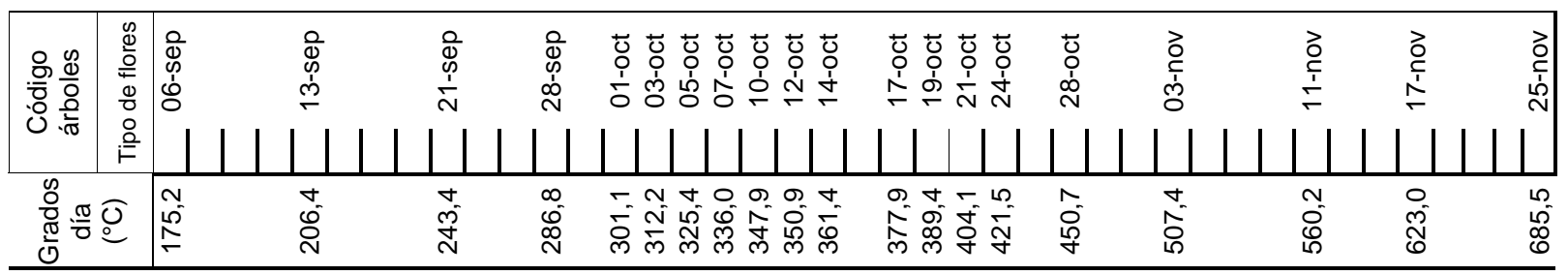

\begin{tabular}{|l|l|l|l|}
\hline 38 & 0 & L. p \\
\hline & P & R \\
\hline
\end{tabular}

Estadío

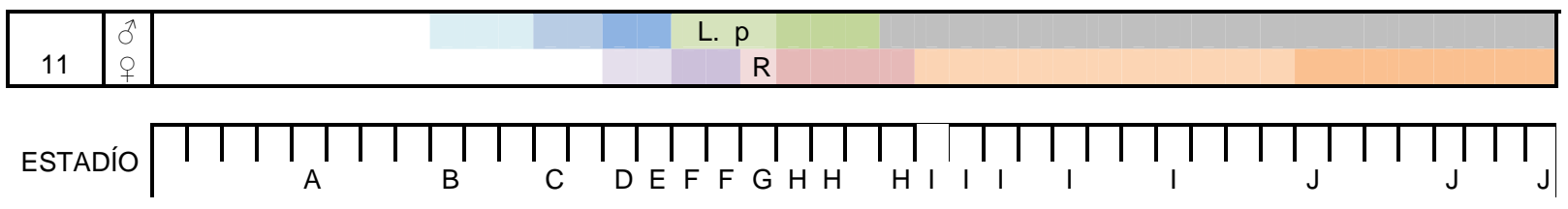

\begin{tabular}{|l|l|l|l|}
\hline & 0 & L. p \\
361 & + & $R$ \\
\hline
\end{tabular}

estadío

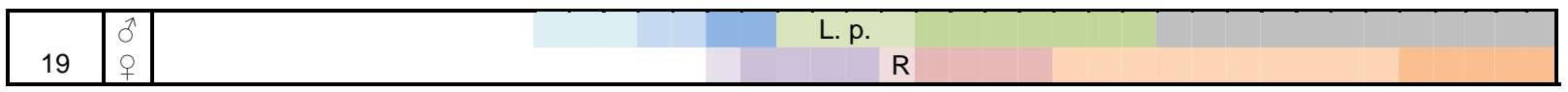

Estadío

\begin{tabular}{|l|l|l|l|}
\hline & 0 & L. p. & \\
\hline & 0 & R & R \\
\hline
\end{tabular}

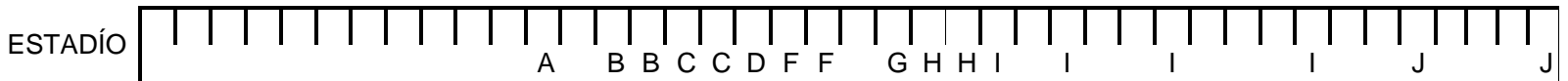

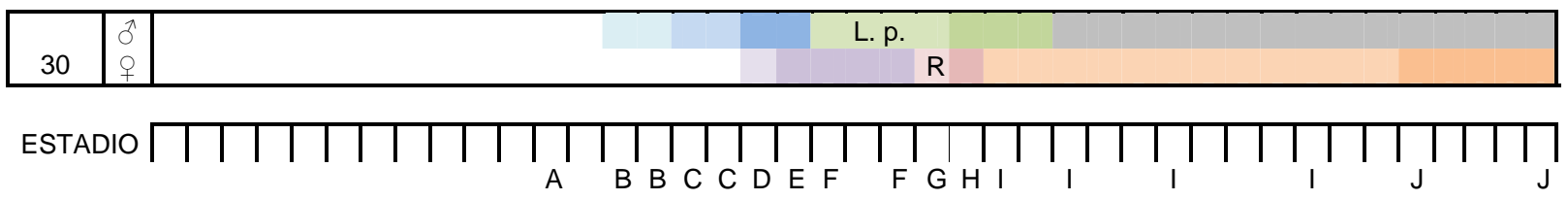

\footnotetext{
*A = yema en latencia, $\mathrm{B}=$ yema hinchada, inicio de apertura de yema - flores masculinas visibles, $\mathrm{C}=$ flor masculina expuesta, $\mathrm{D}=$ periodo de maduración de anteras - inflorescencia femenina visible, $\mathrm{E}=$ anteras maduras - estigmas de flores femeninas en crecimiento, $\mathrm{F}=$ liberación de polen - estigmas de flores femeninas en crecimiento, $G=$ flor masculina senescente con anteras vacías - flores femeninas con estigmas receptivos, $\mathrm{H}=$ caída de flores masculinas - crecimiento de valvas encerrando a los estigmas, I = hinchamiento de ovarios, $\mathrm{J}=$ fruto tierno.
} 
éstas, inmediatamente después de pasar por el periodo de receptibilidad, se observa el inicio del desarrollo de los ovarios, y el crecimiento y la unión de las valvas que encierran progresivamente a los estigmas deshidratados. (figura $2 \mathrm{H}, 2 \mathrm{H}$ ).

- Estado I- hinchamiento de ovarios. Durante este estudio, esta fenofase se observó durante 22 a 26 días (cuadro 4), corresponde al periodo que transcurre desde la fecundación, hasta cuando los frutos completan su desarrollo y alcanzan su máximo tamaño. Los ovarios crecen en las flores que han sido polinizadas o fecundadas; al tiempo que se alarga el pedicelo llegando a medir $0,6 \mathrm{~cm}$ de longitud. Los estigmas aparecen unidos directamente al extremo superior del ovario. Las valvas del involucro, se separan debido al desarrollo del ovario (figura 2I, 2I, 3I).

- Estado J- fruto inicial. Este estado corresponde a la infrutescencia, cuando ha alcanzado su máximo tamaño. La infrutescencia consta de un involucro común en forma de cúpula, de color verde claro, dehiscente, tetrapartida, sus cuatro valvas están provistas de apéndices foliáceos glandulosos. La infrutescencia mide hasta 1,4 cm de diámetro y está sostenida por un pedicelo de hasta $0,6 \mathrm{~cm}$ de longitud (figura $2 \mathrm{~J}$ ).

Duración de los estados florales y requerimiento térmico. El desarrollo de los brotes florales, desde la apertura de la yema hasta el fruto inicial, resultó ser diferente en los clones evaluados. Ocurrió durante un lapso de 58 días en los individuos con brotación temprana y en 40 días en los individuos con brotación tardía. Este crecimiento, calculado en función del calor acumulado, fue de 560,0 a 623,0 grados días de desarrollo, respectivamente (cuadros 4 y 6).

La aparición de la floración masculina, que coincidió con la apertura de los brotes (figuras 2B2, 2B3), se observó en diferentes fechas para los distintos clones evaluados. Desde el 13 de septiembre hasta el 12 de octubre en el individuo más precoz (clon 38), con requerimiento de calor acumulado comprendidos entre 206,4 y 350,9 grados días de desarrollo. El clon 4 y 30 fueron los últimos en presentar la floración, desde el 05 de octubre hasta el 19 de octubre, con un requerimiento de 325,4 a 389,4 grados días de desarrollo (cuadros 4 y 6).

En los clones de origen altitudinal más bajo, las inflorescencias femeninas fueron visibles el 28 de septiembre, fecha en la cual se había acumulado 286,75 grados días de desarrollo y que coincide con el estadio de maduración de anteras en las flores masculinas. En cambio, en los últimos individuos que florecieron, que fueron los clones de mayor origen altitudinal, las inflorescencias femeninas fueron visibles el 10 de octubre, fecha en la que se habían acumulado 347,9 grados días de desarrollo. El tiempo transcurrido, desde el estado de flor visible, hasta flor receptiva fue de 14 días en los individuos de origen altitudinal más bajo y de nueve días en los árboles de mayor altitud. El estado de receptividad de los estigmas en las flores femeninas, se observó el 10 de octubre en los individuos con brotación temprana y el 19 de octubre para los individuos con brotación tardía, lo que calculado en tiempo fisiológico corresponde a 347,9 y 389,4 grados días de desarrollo (cuadros 4 y 6).

Coincidentemente, el inicio de la brotación en los diferentes genotipos evaluados estuvo relacionado al origen altitudinal del ortet. Así, en los clones cuyo lugar de origen se encontraba a menor altitud, los brotes salieron más temprano de la latencia (figura 3 ).

Índice de sobreposición. El índice de sobreposición de la floración entre los seis clones evaluados es bajo $(0,22 \pm$ $0,27)$. Según el traslape de la floración, se destacan tres grupos que tienen buenas posibilidades de cruzarse: un primer grupo consiste en los individuos 38, 11 y $361(0,4$ a 0,67), el segundo grupo formado por los clones 361 y 19 con un índice de solapamiento de 0,67 , y el tercer grupo está formado por los individuos 19, 4 y 30, con un índice de 0,50 (cuadro 5).

\section{DISCUSIÓN}

En N. alpina, al igual que en otras caducifolias leñosas, al iniciar la brotación se presentan simultáneamente hojas

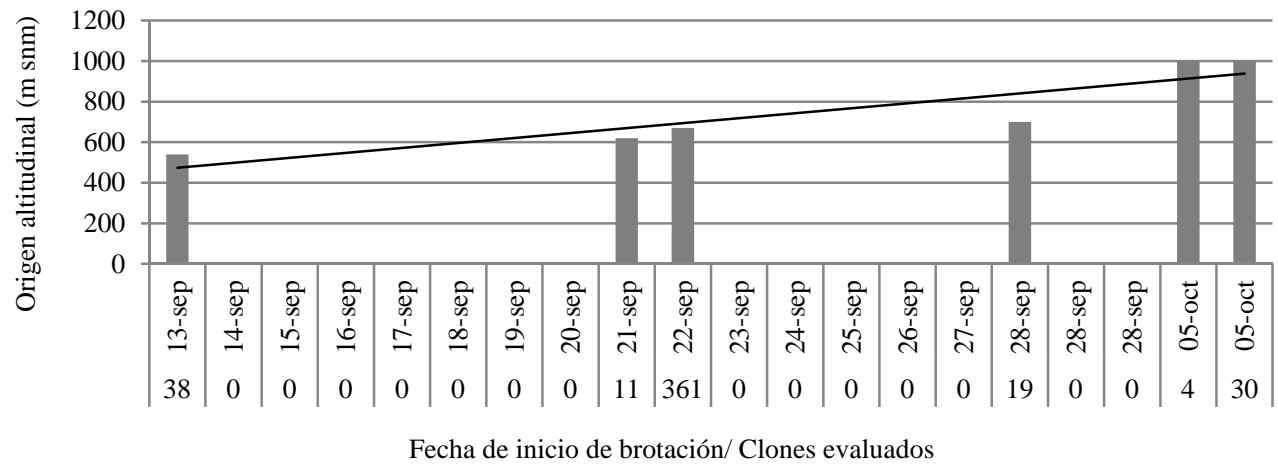

Figura 3. Fecha de inicio de la brotación y origen altitudinal de clones evaluados, huerto semillero Huillilemu, 2011. Starting date of leaf bud breaking and altitudinal origin of clones evaluated, seed orchard Huillilemu, 2011. 
Cuadro 5. Índice de sobreposición de Sörensen de seis clones de Nothofagus alpina en el huerto semillero Huillilemu, 2011. Sörensen index of overlapping of six clones of Nothofagus alpina in the seed orchard Huillilemu, 2011.

\begin{tabular}{|c|c|c|c|c|c|c|c|c|}
\hline & 38 & 11 & 361 & 19 & 4 & 30 & $\bar{X}$ & Sd \\
\hline$\theta^{\lambda}$ & & & & & & & & \\
\hline 38 & 0,4 & 0,4 & 0 & 0 & 0 & 0 & 0,13 & 0,21 \\
\hline 11 & 0,67 & 0,5 & 0,5 & 0 & 0 & 0 & 0,28 & 0,31 \\
\hline 361 & 0 & 0,67 & 0,67 & 0 & 0 & 0 & 0,22 & 0,34 \\
\hline 19 & 0 & 0 & 0,67 & 0,5 & 0 & 0 & 0,19 & 0,31 \\
\hline 4 & 0 & 0 & 0 & 0,5 & 0,5 & 0,5 & 0,25 & 0,27 \\
\hline 30 & 0 & 0 & 0 & 0,5 & 0,5 & 0,5 & 0,25 & 0,27 \\
\hline $\bar{X}$ & 0,18 & 0,26 & 0,31 & 0,25 & 0,17 & 0,17 & 0,22 & 0,27 \\
\hline
\end{tabular}

Cuadro 6. Tiempo fisiológico para las principales fenofases de Nothofagus alpina.

\begin{tabular}{|c|c|c|c|}
\hline Fenofase & Clones & Fecha & $\begin{array}{c}\text { Grados día } \\
\text { acumulados }\end{array}$ \\
\hline \multirow{6}{*}{$\begin{array}{l}\text { Brotación/ } \\
\text { FMV }\end{array}$} & 38 & 13 de septiembre & 206,4 \\
\hline & 11 & 21 de septiembre & 243,4 \\
\hline & 361 & 21 de septiembre & 243,4 \\
\hline & 19 & 28 de septiembre & 286,8 \\
\hline & 4 & 01 de octubre & 301,1 \\
\hline & 30 & 01 de octubre & 301,1 \\
\hline \multirow{6}{*}{$\begin{array}{c}\text { Flor } \\
\text { masculina } \\
\text { expuesta }\end{array}$} & 38 & 21 de septiembre & 243,4 \\
\hline & 11 & 28 de septiembre & 286,8 \\
\hline & 361 & 28 de septiembre & 286,8 \\
\hline & 19 & 03 de octubre & 312,2 \\
\hline & 4 & 05 de octubre & 325,4 \\
\hline & 30 & 05 de octubre & 325,4 \\
\hline \multirow{6}{*}{$\begin{array}{l}\text { Liberación } \\
\text { de polen }\end{array}$} & 38 & 03 de octubre & 312,2 \\
\hline & 11 & 05 de octubre & 325,4 \\
\hline & 361 & 10 de octubre & 347,9 \\
\hline & 19 & 12 de octubre & 350,9 \\
\hline & 4 & 14 de octubre & 361,4 \\
\hline & 30 & 14 de octubre & 361,4 \\
\hline \multirow{6}{*}{$\begin{array}{c}\text { Flor } \\
\text { femenina } \\
\text { visible }\end{array}$} & 38 & 28 de septiembre & 286,8 \\
\hline & 11 & 01 de octubre & 301,1 \\
\hline & 361 & 05 de octubre & 325,4 \\
\hline & 19 & 07 de octubre & 336,0 \\
\hline & 4 & 10 de octubre & 347,9 \\
\hline & 30 & 10 de octubre & 347,9 \\
\hline \multirow{6}{*}{$\begin{array}{c}\text { Flor } \\
\text { femenina } \\
\text { receptiva }\end{array}$} & 38 & 10 de octubre & 347,9 \\
\hline & 11 & 10 de octubre & 347,9 \\
\hline & 361 & 12 de octubre & 350,9 \\
\hline & 19 & 17 de octubre & 377,9 \\
\hline & 4 & 19 de octubre & 389,4 \\
\hline & 30 & 19 de octubre & 389,4 \\
\hline
\end{tabular}

y flores masculinas. Después de la apertura de los catáfilos, hay un rápido desarrollo de los brotes; que consiste en la extensión de órganos preformados en el interior de las yemas. La acelerada elongación de los brotes, depende totalmente de los recursos almacenados en la planta en la estación de crecimiento anterior. Esta característica se considera, junto a la abscisión foliar, determinante en la estrategia para maximizar los beneficios netos de la fotosíntesis (Marti et al. 2004).

La morfología de las flores de $N$. alpina, evidencia su hábito anemófilo. La anemofilia involucra adaptaciones como: flores masculinas con muchos estambres, producción de gran cantidad de granos de polen, largos filamentos y presenta pedúnculos que facilitan su movilidad y flores femeninas poco vistosas, apétalas, con grandes estigmas.

La floración en $N$. alpina, como en otras especies del género, se observó en primavera, entre septiembre y octubre para los clones seleccionados. Esta apreciación visual, considerada en fenología, corresponde solamente a la elongación de brotes, puesto que como en otras caducifolias leñosas, estas flores han sido preformadas en la estación de crecimiento anterior (Marti et al. 2004, Puntieri et al. 2009). Precisamente, los autores de este estudio, han observado la preformación de flores a finales de la primavera en varios árboles de $N$. alpina, en el huerto semillero Huillilemu. La preformación de las flores, es un comportamiento habitual de la especies caducifolias leñosas cuyas yemas son de tipo catafilarias (Martí et al. 2004), como es el caso del N. alpina.

Esta especie presenta dicogamia como estrategia para evitar la autopolinización y de esta forma favorecer la alogamia. La dicogamia observada en la especie es de tipo protándrica. La protandria o liberación anticipada del polen, con respecto a la receptividad del estigma, es una estrategia muy extendida en el reino vegetal, que reduce las oportunidades de autopolinización (Bertin y Newman 1993). La dicogamia no sería el único mecanismo que utiliza esta especie para evitar la autogamia, pues según los estudios de Riveros et al. (1995) las especies de género Nothofagus presentan autoincompatibilidad. 
Coincidentemente, el periodo de floración se acortó en los árboles provenientes de mayor altitud, debido al retraso en el inicio de la brotación. Rusch (1993) y Premoli et al. (2007) realizaron la misma observación al estudiar la fenología de $N$. pumilio (Poepp. et Endl.) Krasser en diferentes condiciones altitudinales en Argentina. El inicio de la brotación también estuvo relacionado al origen altitudinal de los árboles evaluados. Los brotes de los clones cuyo lugar de origen se encontraba a menor altitud salieron más temprano de la latencia. Dicha tendencia, también fue registrada por Rusch (1993) en N. pumilio en Argentina y por Dittmar y Elling (2006) en Fagus sylvatica L., en el Sur de Alemania, en donde se encontró un retraso en las fases fenológicas de dos días en promedio por cada 100 metros de aumento en altitud. Esta tendencia se ha explicado principalmente, como un efecto de diferentes regímenes de temperatura (Rötzer 1996), la radiación y las precipitaciones, que varían con la altitud (Dittmar y Elling 2006).

Dado que en este estudio, si bien se evaluaron genotipos de diferentes orígenes altitudinales, éstos árboles se encuentran bajo las mismas condiciones climáticas en un huerto semillero. Por esta razón, la dependencia entre el inicio de la brotación y el origen altitudinal de los árboles sugiere un control genético de esta característica. En este mismo sentido, Premoli y Mathiasen (2011), concluyeron que existen diferencias genéticas en $N$. pumilio en caracteres ecofisiológicos asociados a la fenología, crecimiento, fotosíntesis y morfología foliar. Estos autores, también mencionan que estas diferencias habrían surgido como resultado de presiones de selección, reforzadas por un flujo génico limitado por el desajuste de la fenología, entre poblaciones distanciadas pocos cientos de metros en el gradiente altitudinal. En el huerto semillero Huillilemu, el retraso de esta fenofase es más evidente (un promedio de nueve días por cada 100 metros de aumento en la altitud), esto se debe, posiblemente, a que sumado a la predisposición genética exista una posible falta de requerimiento de frío, pues se sabe que una planta que no ha completado satisfactoriamente sus requerimientos de frío, necesita mayor acumulación de calor para salir de la dormancia invernal, lo que conlleva un retraso en la brotación (Erez 1987). Aunque esta tendencia de atraso en la brotación y acortamiento del periodo de floración, asociada al origen altitudinal de los clones analizados, resulta bastante sugerente, debería estudiarse una mayor cantidad de clones por clase de altitud, para corroborar la hipótesis.

Además, debido a que los caducifolios, después de satisfacer sus requerimientos de frío, necesitan simultáneamente calor para activar los procesos de polinización y de formación de las flores y frutos; se cuantificaron los grados día requeridos en el desarrollo del brote floral, desde la apertura hasta el fruto inicial. El calor acumulado para el desarrollo de los brotes florales, varió en los diferentes clones, lo que indica que cada genotipo tiene su propio requerimiento de grados día.
El índice general de sobreposición de la floración para los clones evaluados es bajo, lo que pone en relieve la baja sincronía reproductiva que tendría la población evaluada; pues las diferencias en la fenología, no permitirían el intercambio de genes vía polen entre los genotipos establecidos en el huerto. Según Premoli y Mathiasen (2011), las diferencias fenológicas en las poblaciones vegetales a lo largo de gradientes altitudinales, causarían su aislamiento reproductivo.

Además de la diferencia en las fechas de floración, habría que analizar otros aspectos que podrían limitar la producción de semillas viables en el huerto semillero Huillilemu, como la viabilidad y longevidad del polen, así como posibles condiciones ambientales desfavorables que limitarían una polinización efectiva, como la dirección y velocidad del viento, humedad relativa y temperatura. En este aspecto, Franquel y Galum (1977) sostienen que la humedad relativa baja y la temperatura alta son favorables para un buen flujo de polen. También, los mismos árboles que componen la plantación forman una barrera que impide el libre flujo de polen. Así mismo, se debe considerar, que aunque el índice de sobreposición de las flores en un mismo clon es alto, no se espera que suceda autofertilización, pues se ha documentado autoincompatibilidad en otras especies del género Nothofagus (Riveros et al. 1995).

\section{CONCLUSIONES}

Nothofagus alpina se caracteriza por una marcada estacionalidad reproductiva, cuyo periodo de floración se desarrolla en la primavera. La morfología floral de N. alpina, es característica de las especies de polinización anemófila. Los cambios progresivos desde la yema latente hasta la presencia del fruto tierno se resumen en 10 estados fenológicos. Se determinó dicogamia en forma de protandria, en esta especie, pues se observó una liberación temprana del polen con respecto a la receptividad del estigma. La fecha de inicio de la brotación, está fuertemente relacionada al origen altitudinal de los clones evaluados, por lo que se sugiere que esta característica estaría bajo control genético. El índice general de sobreposición es de 0,22, que indica que debido al desfase en la fenología y el corto periodo de receptibilidad de las flores femeninas, las posibilidades de cruzamiento entre los clones evaluados son bajas.

\section{REFERENCIAS}

Bertin RI, CM Newman. 1993. Dichogamy in angiosperms. Botanical Review 59: 112-152.

De Fina AL, AC Ravelo. 1979. Climatología y Fenología Agrícolas. Buenos Aires, Argentina. Tercera Edición. Editorial Universitaria de Buenos Aires. 359 p.

Erez A. 1987.Chemichal control of budbreak. Hortscience 22(6): 1240-1243.

Dittmar C, W Elling. 2006. Phenological phases of common beech (Fagus sylvatica L.) and their dependence on region and altitude in Southern Germany. European Journal of Fo- 
rest Research 125: 181-188.

Donoso PJ, C Donoso, M Azpiculeta. 2006. Especies arbóreas de los bosques templados de Chile y Argentina. Autoecología. Valdivia, Chile. Marisa Cúneo Ediciones. 678 p.

Gómez-Casero MT, C Gálan, E Domínguez-Vilches. 2007. Flowering phenology of Mediterranean Quercus species in different locations (Córdoba, SW Iberian Peninsula). Acta Botanica Malacitana 32: 127-146.

Hill RS, GJ Jordan. 1993. The evolutionary history of Nothofagus (Nothofagaceae). Australian Systematic Botany 6: 111-126.

Köppen W. 1900. Versuch einer Klassifikation der Klimate, vorzugsweise nach ihren Beziehungen zur Pflanzenwelt. Geographische Zeitschrift 6: 593-611, 657-679.

Lieth H. 1997. Aims and methods in phenological monitoring, in Phenology. In Lieth H, MD Schwartz ed. Seasonal Climates. Leiden, The Netherlands. p. 1-21.

Martí MG, S Palacio, R Milla. 2004. Fenología y características funcionales de las plantas leñosas mediterráneas. In Valladares $\mathrm{F}$ ed. Ecología del bosque mediterráneo en un mundo cambiante. Madrid, España. Ministerio de Medio Ambiente, EGRAF. p. 129-162.

Premoli AC, E Raffaele, P Mathiasen. 2007. Morphological and phenological differences in Nothofagus pumilio from contrasting elevations. Austral Ecology 32: 515-523.

Premoli AC, P Mathiasen. 2011. Respuestas ecofisiológicas adaptativas y plásticas en ambientes secos de montaña: Nothofagus pumilio, el árbol que acaparó los Andes Australes. Ecología Austral 21: 251-269.

Puntieri JG, JE Grosfeld, P Heuret. 2009. Preformation and distribution of staminate and pistillate flowers in flowers in growth units of Nothofagus alpina and N. obliqua (Nothofagaceae). Annals of Botany 103: 411-421.

Puntieri JG, JE Grosfeld, M Stecconi, C Brion, D Arthelemy. 2007. Bud and growth-unit structure in seedlings and
Saplings of Nothofagus alpina (Nothofagaceae). American Journal of Botany 94(8): 1382-1390.

Riveros M, MA Paredes, MT Rosas, E Cardenas, JJ Armesto, M Arroyo. 1995. Reproductive biology in species of the genus Nothofagus. Environmental and Experimental Botany 35: 519-524.

Rozefelds A, A Drinnan. 1998. Ontogeny and diversity in staminate flowers of Nothofagus (Nothofagaceae). International Journal of Plant Sciences 159(6): 906-922.

Rozefelds A, A Drinnan. 2002. Ontogeny of pistillate flowers and inflorescences in Nothofagus subgenus Lophozonia (Nothofagaceae). Plant Systematics and Evolution 233: 105-126.

Rusch VE. 1993. Altitudinal variation in the phenology of Nothofagus pumilio in Argentina. Revista Chilena de Historia Natural 66: 131-141.

Rötzer T. 1996 Neuartige Karten der Phänologie und des Wasserhaushaltes von Bayern unter Berücksichtigung möglicher künftiger Klimaverhältnisse. Dissertation an der TU München Weihenstephan. $182 \mathrm{p}$.

Schwartz MD. 1999. Advancing to full bloom: planning phenological research for the twenty-first century. International Journal of Biometeorology 42: 113-118.

Sistema Agroclimático FDF-INIA-DMC. 2012 Informes agroclimáticos, Región de los Ríos. Consultado 23 oct. 2012. Disponible en www.agroclima.cl

Souza L, K Mourão, I Moscheta, S Rosa. 2003. Morfologia e anatomia da flor de Pilocarpus pennatifolius Lem. (Rutaceae). Revista Brasileira de Botânica 26(2): 175-184.

Urban O. 1934. Botánica de plantas endémicas de Chile. Concepción, Chile. 291 p.

Sörensen TA. 1948. A method of establishing groups of equal amplitude in plant sociobiology based on similarity of species content and its application to analyses of vegetation in Danish commons. Biologiske Skrifter 5: 1-40. 
